\title{
THE HYDRODYNAMIC EFFICIENCY OF LASER-TARGET ACCELERATION
}

\author{
J. R. Sanmartín, J. L. Montañés, J. Sanz and R. Ramis \\ Escuela Técnica Superior de Ingenieros Aeronáuticos, 28040 Madrid, Spain
}

\begin{abstract}
The acceleration of a thin foil using a laser pulse is studied. It is shown that the acceleration efficiency $\eta_{H}$ is heavily dependent on the behaviour of the corona ejected by the foil: there is no universal relation $\eta_{H}\left(\Delta M / M_{0}\right), M_{0}$ and $\Delta M$ being initial foil mass and ablated mass, respectively. Known results on the coronal flow are used to check the theory against experimental data available in the literature; effects due to both a non-planar corona, and the time-dependence of the laser irradiance, are considered. The agreement with experiments is substantially better than that for previous analyses. Acceleration of thin spherical shells is also discussed.
\end{abstract}

\section{INTR ODUCTION}

ONE of the most important parameters in laser-fusion is the fraction $\eta$ of absorbed laser energy $E_{a}$ that goes into the target. In experiments on acceleration of thin foils (or thin spherical shells), most of the final energy within the remains of the target may be kinetic energy. Then $\eta$ is the hydrodynamic efficiency, $\eta_{H}=M u^{2} / 2 E_{a}$, where $M$ is the unablated mass and $u$ its velocity, assumed uniform throughout it. Usually (MCCALL, 1983; RIPIN et al., 1980; DUdERSTADT and Moses, 1982) some simple rocket model is used to calculate $\eta_{H}$. The standard formula for $\eta_{H}$ is

$$
\eta_{I}=\frac{\left(\ln \left(1-\Delta M / M_{0}\right)\right)^{2}\left(1-\Delta M / M_{0}\right)}{\Delta M / M_{0}} \equiv \bar{\eta}\left(\frac{\Delta M}{M_{0}}\right),
$$

$M_{0}$ and $\Delta M$ being initial and ablated mass. Some corrections to (1) have been discussed in the past (FABBRO, 1982; MAX et al., 1983).

Here we reconsider the calculation of $\eta_{H}$. We show that when known results on the expanding plasma (in the corona outside the ablation surface) are taken into account, $\eta_{H}$ differs substantially from previously published values. In the paper we also discuss time-dependent and non-planar effects on the hydrodynamic efficiency. On the whole, the agreement with experimental data is greatly improved.

In Section 2 we review the analysis of planar coronae. In Section 3 both steady and unsteady planar-target acceleration are considered. Section 4 studies non-planar effects on acceleration, making use of known results on spherical coronae. Section 5 collects conclusions from all previous sections to compare theory with experimental data on $\eta_{H}$ available in the literature. Spherical shells are discussed in the Appendix.

\section{THE ANALYSIS OF A PLANAR CORONA}

Let laser-light be incident on a solid foil on the left, and the beam cross-section be large enough to allow considering the problem as one-dimensional (Fig. 1). Under broad conditions the equations for the quasineutral plasma ablated from the target 


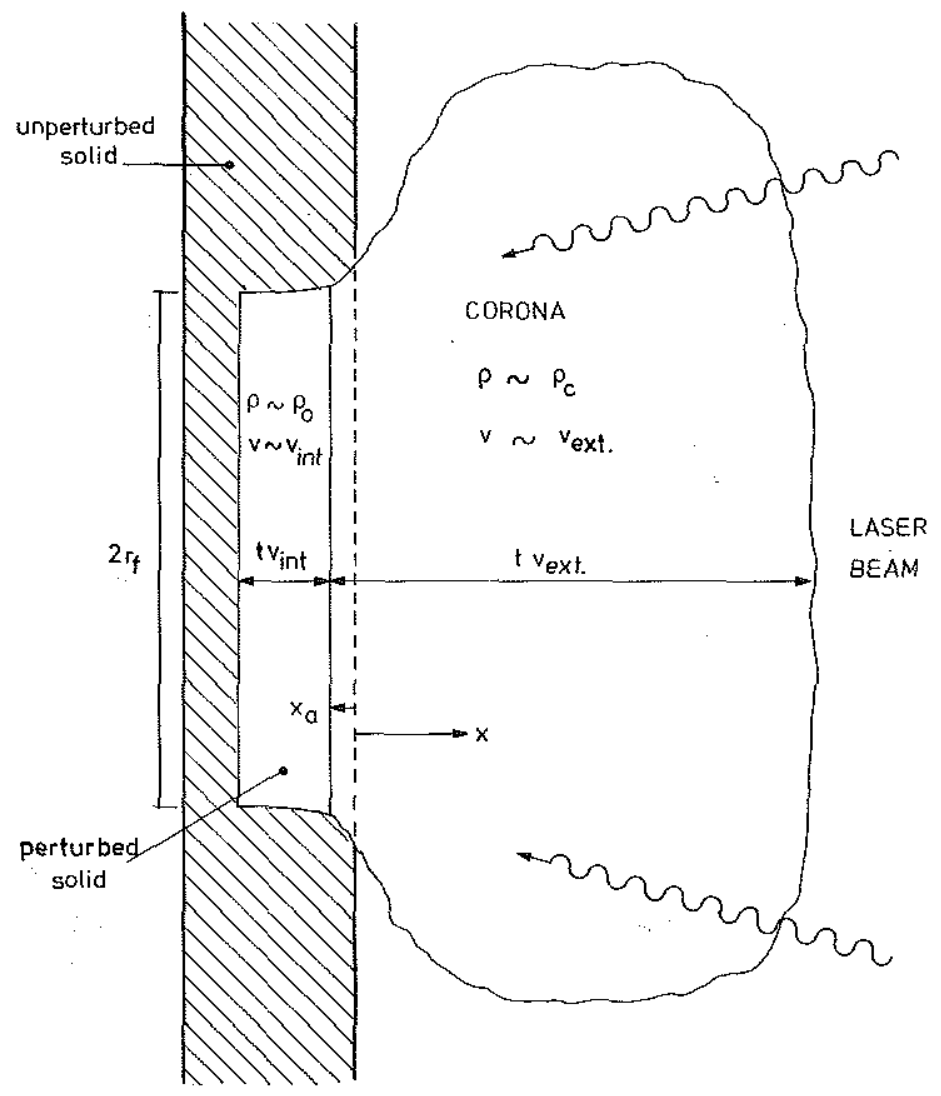

FIG. 1.-Interaction between a laser-beam incident from the right and a solid foil, before the perturbation reaches its back: $r_{f}$ is the spot radius, $\rho_{\mathrm{c}}$ and $\rho_{0}$ are critical and unperturbed solid densities, respectively; $x_{a}$ locates the ablation surface.

are conservation laws for mass, momentum, and energy, in the form

$$
\begin{gathered}
\frac{\partial \rho}{\partial t}+\frac{\partial}{\partial x} \rho v=0 \\
\frac{\partial}{\partial t} \rho v+\frac{\partial}{\partial x}\left(\rho v^{2}+P\right)=0 \\
\frac{\partial}{\partial t} \rho\left(e_{i n}+\frac{1}{2} v^{2}\right)+\frac{\partial}{\partial x}\left(\rho v\left(e_{i n}+\frac{1}{2} v^{2}\right)+v P+q+I_{+}-I_{-}\right)=0
\end{gathered}
$$

and the ion entropy equation

$$
\rho T_{i}\left(\frac{\partial}{\partial t}+v \frac{\partial}{\partial x}\right) s_{i}=\frac{3}{2} n \frac{T_{e}-T_{i}}{t_{e_{i}}}
$$


The mass density above is $\rho=\bar{m} n \equiv m_{i} n / Z_{i}$, where $n$ is electron density. The pressure is $P=n T_{e}+n T_{i} / Z_{i}$; the specific internal energy and ion entropy are $e_{i n}=3 T_{e} / 2 \bar{m}+$ $3 T_{i} / 2 \bar{m} Z_{i}$, and $s_{i}=m_{i}^{-1} \ln \left(T_{i}^{3 / 2} / n\right)+$ const. The heat flux is $q=\min \left(\bar{K} T_{e}^{5 / 2}\left|\mathrm{~d} T_{e} / \mathrm{d} x\right|\right.$, $\left.f n T_{e}^{3 / 2} m_{e}^{-1 / 2}\right) \times \operatorname{sign}\left(-\mathrm{d} T_{e} / \mathrm{d} x\right), \tilde{K} T_{e}^{5 / 2}$ being Spitzer's conductivity (SPITZER, 1967) and $f$ a flux-saturation factor (DUDERSTADT and MOSES, 1982); the ion-electron energy relaxation time $t_{e_{i}} \propto m_{i} \bar{K} T_{e}^{3 / 2 /} / n$ is also given by Spitzer. The incident and reflected irradiances $I-$ and $I_{+}$change according to $\partial I_{\mp} / \partial x= \pm \kappa I_{\mp}\left(x>x_{c}\right)$ where $\kappa$ is the absorption coefficient due to inverse bremsstrahlung (JOHNSTON and DAwsON, 1973); also $I_{+}\left(x_{c}^{+}\right)=(1-\alpha) I_{-}\left(x_{c}^{+}\right)$, $\alpha$ being an anomalous absorption fraction (ForsLUND et al., 1977; EsTABROOK and KRUER, 1978) and $x_{c}$ such that $n\left(x_{c}\right)=n_{c} \equiv$ critical density. As $x \rightarrow+\infty$, one has $I_{-} \rightarrow I_{L}(t)$ (the laser-pulse irradiance, characterized by a peak value $I_{m}$ and a full-width at half-maximum $\tau$ ).

Two facts simplify the problem of solving analytically system (2)-(5) to yield $n, v$, $T_{e}$, and $T_{i}$ as functions of $x$ and $t$. Usually the density in the perturbed solid (a few times $\left.\rho_{0}\right)$ is large compared with the characteristic density $\left(\rho_{c}\right)$ in the corona, which is the region to the right of the (ablation) surface $x=x_{a}(t)$ where $v \simeq 0, \partial P / \partial x \simeq 0$. For instance, $\rho_{0} / \rho_{c} \simeq 50$ for solid $D-T$ and $1.06 \mu \mathrm{m}$ light; $\rho_{0} / \rho_{c}$ is much larger for targets of high atomic number, for which $Z_{i} \gg 1$. In analysing the corona we might tentatively set $\rho_{0} / \rho_{c} \rightarrow \infty$. This would require that $\rho \rightarrow \infty$ as $x \rightarrow x_{a}(t)$, leading to $T_{e}, T_{i} \rightarrow 0$ there, because mass and momentum within the corona, and thus surface mass ablation rate $\dot{m}_{s}$ and ablation pressure $P_{a}$, must remain finite. The fact that makes possible such behaviour $\left(v, T_{e}, T_{i}\right.$ vanishing at a surface which is at finite distance from a region where energy is deposited) is the nonlinear character of plasma heat conduction. Nonlinear heat waves in the absence of motion have been studied extensively (ZEL'DOVICH and RAIZER, 1967).

For a thick foil or a short pulse, the perturbed mass per unit surface in (the irradiated part of) the foil is of order $t v_{\text {int }} \rho_{0}$ (Fig. 1); $v_{\text {int }}$ and $v_{\text {ext }}$ are characteristic velocities for corona and perturbed solid. Conservation of momentum yields

$$
v_{\text {int }} \times t v_{\text {int }} \rho_{0} \sim v_{\text {ext }} \times t v_{\text {ext }} \rho_{c} \sim v_{\text {ext }} \times \Delta M_{s},
$$

where $\Delta M_{s}$ is the surface ablated mass. Then

$$
\left|\dot{x}_{a}\right| \sim v_{\text {int }} \ll v_{\text {ext }} \rightarrow x_{a}(t) \simeq x_{a}(0)=0 .
$$

Equation (7) completes the uncoupling of corona and target; all is needed to analyse the corona is that it starts at $x=0$, and that is hot and rarefied when compared with the target.

For the thin foils of interest here we have $t v_{\mathrm{int}} \rho_{0}>M_{s}$ (surface mass in the target), so that (6) should read

$$
v_{\text {int }} \times M_{s} \sim v_{\text {ext }} \times \Delta M_{s} ;
$$

thus condition (7) requires $\Delta M_{s} / M_{s}$ to be small (Section 3). 
In writing down dimensionless results from the analysis, it proves convenient to introduce a characteristic speed

$$
\begin{aligned}
U & \equiv\left(n_{\mathrm{c}} \tau / \bar{m}^{5 / 2} \bar{K}\right)^{1 / 3} \\
& \simeq 0.915 \times 10^{7} \frac{\mathrm{cm}}{\mathrm{s}}\left(\frac{2 Z_{i}}{A_{i}}\right)^{5 / 6}\left(\frac{1.06 \mu \mathrm{m}}{-\lambda}\right)^{2 / 3}\left(\frac{\tau}{1 \mathrm{~ns}} \frac{Z_{i} \ln \Lambda}{100 \varepsilon \delta_{T}}\right)^{1 / 3}
\end{aligned}
$$

which substitutes the awkward factor $\bar{K}$ in the set of dimensional parameters available. Quantities of interest, such as peak values of $P_{a} / \rho_{c} U^{2}, \dot{m}_{s} / \rho_{c} U$, are found to be functions of five dimensionless numbers: $\alpha, Z_{i}, f\left(\bar{m} / m_{e}\right)^{1 / 2}, \hat{I}_{m} \equiv I_{m} / \rho_{c} U^{3}$, and $\hat{U} \equiv \bar{m} U / m_{e} c .\left[\hat{I}_{m}\right.$ characterizes the steepness of the pulse, $\hat{U}$ bremsstrahlung absorption; note that $\kappa \propto n^{2} / \bar{K} m_{e} c n_{c}\left(1-n / n_{c}\right)^{1 / 2} T_{e}^{3 / 2}$.] Fluid variables depend, in addition, on $t / \tau$ and $x / U \tau$. For specific power-law pulses, $I_{L} \propto t$ (ANISIMov, 1970; BarRero and SANMARTIN, 1977), $I_{L} \propto t^{3 / 2}$ (NICOLAS, 1984), the flow may be self-similar and has been analysed in detail in the past (SANMARTIN and BARRERo, 1978a, b; BARrero and SANMARTIN, 1980; Ramis and SaNMARTIN, 1983; NiCOLAS, 1984).

\section{PLANAR TARGET ACCELERATION}

If $M_{s}$ is the mass per unit area remaining in the target at any given time, and $u$ its velocity, then (see Appendix A)

$$
\begin{aligned}
\mathrm{d} M_{s} / \mathrm{d} t & =-\dot{m}_{s} \\
M_{s} \mathrm{~d} u / \mathrm{d} t & =-P_{a} .
\end{aligned}
$$

The target experiences a leftward acceleration. To obtain $\eta_{H} \equiv M_{s} u^{2} / 2 \int_{0}^{t} I_{a}\left(t^{\prime}\right) \mathrm{d} t^{\prime}$ we solve (10) and (11) for $t\left(\Delta M_{s}\right), u\left(\Delta M_{s}\right)$ using coronal results for $\dot{m}_{s}$ and $P_{a}$; here $I_{a}$ is absorbed intensity, and $\Delta M_{s} \equiv M_{s 0}-M_{s}$.

First neglect inverse bremsstrahlung $(\hat{U} \rightarrow 0)$ and consider the linear pulse $I_{a}=\alpha I_{L} \equiv I_{a m} t / \tau$. In the resulting self-similar motion one gets

$$
\dot{m}_{s}=\dot{m}_{s m}(t / \tau)^{1 / 3}, P_{a}=P_{a m}(t / \tau)^{2 / 3} .
$$

One may then integrate (10) and (11) to obtain

$$
\eta_{H}=\frac{P_{a m}^{2}}{2 \dot{m}_{s m} I_{a m}} \times \bar{\eta}_{1}\left(\Delta M_{s} / M_{s 0}\right)
$$

where

$$
\bar{\eta}_{1}(\Delta) \equiv \frac{3}{2} \frac{1-\Delta}{\Delta^{3 / 2}}\left(2 \tan ^{-1} \Delta^{1 / 4}+\ln \frac{1+\Delta^{1 / 4}}{1-\Delta^{1 / 4}}-4 \Delta^{1 / 4}\right)^{2} .
$$

The function $\bar{\eta}_{1}(\Delta)$ is close to that appearing in (1)

$$
\bar{\eta}(\Delta) \equiv \frac{1-\Delta}{\Delta}(\ln (1-\Delta))^{2}
$$


the ratio $\bar{\eta}_{1} / \bar{\eta}$ is 0.96 for $\Delta \rightarrow 0,1$ at $\Delta \simeq 0.3$ and 1.17 at $\Delta=0.9$.

Note that $P_{a}^{2}(t) / 2 \dot{m}_{s}(t) I_{a}(t)=\mathrm{const}=P_{a m}^{2} / 2 \dot{m}_{s m} I_{a m}$. The ratio $P_{a}^{2} / 2 \dot{m}_{s} I_{a}$ depends on $Z_{i}, f\left(\bar{m} / m_{e}\right)^{1 / 2}$, and $\hat{I}_{a m}$, though not on $\alpha$. For $\hat{I}_{a m}$ small the corona separates into a quasisteady, thin (deflagration) layer where absorption and conduction occur, and a large region of isentropic expansion. For classical conduction, i.e. $f\left(\bar{m} / m_{e}\right)^{1 / 2}$ large, one gets $P_{a}^{2} / 2 \dot{m}_{s} I_{a}=16 / 25=0.64$ independently of $Z_{i}$ (though both $P_{a}$ and $\dot{m}_{s} d o$ depend on $Z_{i}$ ) (SANMartin and Barrero, 1978a). For $Z_{i} \gg 1$, flux-saturation does not modify this result: $P_{a}^{2} / 2 \dot{m}_{s} I_{a} \simeq 0.64$ for $f>0.03$ (RAMIS and SANMARTIN, 1983).

Results for $\hat{I}_{a m} \gg 1$ and $Z_{i}$ arbitrary, and $Z_{i} \gg 1$ and $\hat{I}_{a m}$ arbitrary, with classical conduction, are known (SANMARTIN and BARRERO, 1978b; BARRERo and SANMARTIN, 1980). For $\hat{I}_{a m}$ and $Z_{i}$ large we have $P_{a}^{2} / 2 \dot{m}_{s} I_{a} \simeq 0.265$. Flux-saturation yields a similar result if $f \simeq 0.6$, and $P_{a}^{2} / 2 \dot{m}_{s} I_{a} \simeq 0.09$ if $f \simeq 0.03$.

When both conduction and inverse bremsstrahlung are considered the flow can not be self-similar. However for small $\hat{I}_{a m}$ conduction only counts in the deflagration layer, which is quasisteady; to make the outside flow self-similar with bremsstrahlung absorption included $[\hat{U}=0(1)]$, we take $I_{L}=I_{m}(t / \tau)^{3 / 2}$ (NICOLAS, 1984). Then one has

$$
\dot{m}_{s}=\dot{m}_{s m}(t / \tau)^{1 / 2}, \quad P_{a}=P_{a m} t / \tau,
$$

and integration of (10) and (11) yields

$$
\eta_{H}=\frac{P_{a m}^{2}}{2 \dot{m}_{s m} I_{a m}} \times \bar{\eta}_{2}\left(\Delta M_{s} / M_{s 0}\right)
$$

where $P_{a m}^{2} / 2 \dot{m}_{s m} I_{a m}=P_{a}^{2}(t) / 2 \dot{m}_{s}(t) I_{a}(t)$, and

$$
\bar{\eta}_{2}(\Delta) \equiv \frac{5}{3} \frac{1-\Delta}{\Delta^{5 / 3}}\left(3^{1 / 2} \tan ^{-1} \frac{3^{1 / 2} \Delta^{1 / 3}}{2+\Delta^{1 / 3}}+\frac{\ln \left(1+\Delta^{1 / 3}+\Delta^{2 / 3}\right)^{1 / 2}}{1-\Delta^{1 / 3}}-3 \Delta^{1 / 3}\right)^{2}
$$

and $I_{a m}=A_{x} I_{m}, A_{T}$ being total absorption; $\bar{\eta}_{2}$ is always very close to $\bar{\eta}_{1}: \bar{\eta}_{2} / \bar{\eta}_{1} \simeq 0.98$ for $\Delta \rightarrow 0$, and 1.03 at $\Delta=0.9$. For $Z_{i}$ and $f\left(\bar{m} / m_{e}\right)^{1 / 2}$ large, $\left(\hat{I}_{a m}\right.$ small $)$, and $\hat{U}=0(1)$ we have $P_{a}^{2} / 2 \dot{m}_{s} I_{a} \simeq 0.314$ independently of $\alpha$; for $\hat{U} \rightarrow 0$ we again have $P_{a}^{2} / 2 \dot{m}_{s} I_{a}=$ 0.64 . Note that inverse bremsstrahlung is negligible for $\hat{I}_{a m}$ large.

In the past, authors always considered $I_{a}=$ const, assuming $P_{a}$ and $\dot{m}_{s}$ constant too. Then equations (10) and (11) give

$$
\eta_{H}=\frac{P_{a}^{2}}{2 \dot{m}_{s} I_{a}} \times \bar{\eta}\left(\frac{\Delta M_{s}}{M_{s 0}}\right)
$$

Either a cold-rocket model $\left(P_{a}^{2} / 2 \dot{m}_{s} I_{a}=1\right)$, or a coronal model consisting of a steady deflagration layer and a self-similar expansion, was used. However, a steady deflagration will only exist if (a) $\hat{I}_{a}(t) \ll 1$, i.e. $t \gg I_{a} \bar{m}^{5 / 2} \bar{K} \rho_{c} n_{c}$; and if (b) $\hat{U}(t) \ll 1$, i.e. $t \ll\left(m_{e} c / \bar{m}\right)^{3} \bar{m}^{5 / 2} \bar{K} / n_{c}$. In the above we used $U(t) \equiv\left(n_{c} t / \bar{m}^{5 / 2} \bar{K}\right)^{1 / 3}$. Condition (a) marks the time required to set up the deflagration; condition (b) means that bremsstrahlung absorption outside the layer will prevail in the long run because the self-similar expansion is growing all the time. These conditions are rarely satisfied simultaneously. If they are satisfied, nonetheless, the expansion will be definitely isentropic, and not 
isothermal (SANMARTIN et al., 1983); this point has produced some confusion in the past.

The result $\bar{\eta} \simeq \bar{\eta}_{1}\left(\simeq \bar{\eta}_{2}\right)$ shows that the shape of the pulse has a negligible effect on the dependence of $\eta_{H}$ on $\Delta M_{s} / M_{s 0}$ (specially so if $\Delta M_{s} / M_{s 0}<0.5$ ). Thus we can use equation (14) for $\eta_{H}$ for a real pulse (which only in its rising-half can be reasonably approximated by a linear pulse). The factor $P_{a}^{2} / 2 \dot{m}_{s} I_{a}$ in equation (14) is to be taken from the above results for the different regimes [depending on the values of $\hat{I}_{m}$, $f\left(\bar{m} / m_{e}\right)^{1 / 2}$, and $\hat{U}$, mainly]. Table 1 resumes those results.

TABLE 1.-VALUes of $P_{a}^{2} / 2 \dot{M}_{s} I_{a}$ IN EQUATION (14) FOR DIFFerent Regimes OF A PlanAr CORONA

\begin{tabular}{llllll}
\hline Regime & $\frac{I_{m}}{\rho_{\mathrm{c}} U^{3}}$ & $\frac{\bar{m} U}{m_{e} c}$ & $f\left(\frac{\bar{m}}{m_{e}}\right)^{1 / 2}$ & $Z_{i}$ & $\frac{P_{a}^{2}}{2 \dot{m} I_{a}}$ \\
\hline I & Small & Small & Large/any & Any/large & 0.64 \\
II & Small & $\sim 1$ & Large & Large & 0.31 \\
III & Large & Small & Large & Large & 0.27 \\
IV & Large & Small & $\simeq 1.8$ & Large & 0.09 \\
\hline
\end{tabular}

The uncoupled analysis of the corona was partly based on the approximation $x_{a}(t) \simeq 0$, and this required $\Delta M_{s} / M_{s 0}$ to be small. For this range we may set $\bar{\eta} \simeq \Delta M_{s} / M_{s 0}$. The analysis should be actually valid up to about $\Delta M_{s} / M_{s 0} \simeq 0.2$ or 0.3 , because (i) the ablated mass is growing during the entire pulse, $\Delta M_{s}$ being its final value, and (ii) $x_{u}(t)$ and $u(t)$ are found from time-integrations.

If $\hat{I}_{a m}$ is small, results may be extended to values $\Delta M_{s} / M_{s 0}=0(1)$. Use of new variables $x^{\prime} \equiv x-x_{a}, v^{\prime} \equiv v-\dot{x}_{a}$, may always take back the problem to the $x_{a}=0$ case, but equations (3) and (4) must then include an inertial force per unit volume, $-\rho \ddot{x}_{a}$, which in general destroys the self-similar character of the flow. This however is unimportant for $\hat{I}_{a m} \ll 1$ because the relation between $\dot{m}_{s}$, or $P_{a}$, and $I_{a}$ is then obtained from an analysis of the deflagration layer, which is steady or quasisteady. Inertial forces have been often considered in the past in the analysis of deflatrations (FELBER, 1977; FABBRO, 1982). In fact, under deflagration conditions the inertial force may be ignored altogether because $-\rho \ddot{x}_{a} \sim \rho P_{a} / M_{s} \sim\left(\Delta M_{s} / M_{s}\right) P_{a} /$ (characteristic length of corona), which is small compared with the sharp-pressure gradient within the deflagration layer (unless $M_{s} / M_{s 0} \ll 1$ ).

\section{TARGET ACCELERATION FOR A NON-PLANAR CORONA}

The assumption of planar geometry for the corona (Section 2) is valid if $\tau v_{\text {ext }} \ll r_{f}$ where $r_{f}$ is the focal spot radius. This condition applies when the pulse is short or the beam is wide. When $\tau v_{\text {ext }} / r_{f}$ is of order unity the coronal flow depends clearly on $Z_{i}, f\left(\bar{m} / m_{e}\right)^{1 / 2}, \alpha, \hat{I}_{m}$, and $\hat{U}$, and on $U \tau / r_{f}$. For long pulses or narrow beams $\left(\tau v_{\text {ext }} \gg r_{f}\right)$ the flow approaches the conditions of spherical geometry. To take this into account we use known results about spherical coronae. In the past the divergence in the flow was dealt with by introducing some mean divergence angle into the coldrocket model (RIPIN et al., 1980).

A discussion similar to that in Section 2 shows that in spherical geometry there exists an ablation surface at some radius $r_{a}(t)$, and that in analysing the corona one may set $v=T_{e}=T_{i}=0, n \rightarrow \infty$, at $r=r_{a}$, and $\dot{r}_{a} \simeq 0$. If, in addition, $W_{L} / \dot{W}_{L} \sim \tau \gg$ 
$r_{a} / v_{\text {ext }}$, the corona may be studied by using a quasisteady approximation (AFANAS'EV et al., 1977; GITOMER et al., 1977; MAX et al., 1980; SANZ et al., 1981); $\tau$ is then an ignorable parameter. One may introduce a characteristic speed that does not involve $\tau$,

$$
V \equiv\left(\frac{n_{c} r_{a}}{\bar{m}^{5 / 2} \bar{K}}\right)^{1 / 4} \simeq 1.40 \times 10^{7} \frac{\mathrm{cm}}{\mathrm{s}}\left(\frac{2 Z_{i}}{A_{i}}\right)^{5 / 8}\left(\frac{1.06 \mu \mathrm{m}}{\lambda}\right)^{1 / 2}\left(\frac{2 r_{a}}{\left.1 \mathrm{~mm} \frac{Z_{i} \ln \Lambda}{100 \varepsilon \delta_{T}}\right)^{1 / 4}}\right.
$$

note that $V=U\left(r_{a} / U \tau\right)^{1 / 4}$. Quantities such as $P_{a} / \rho_{c} V^{2}, \dot{m} / 4 \pi r_{a}^{2} \rho_{c} V$ are then functions of $Z_{i}, f\left(\bar{m} / m_{e}\right)^{1 / 2}, \alpha, \hat{V} \equiv \bar{m} V / m_{e} c$, and $\hat{W} \equiv W_{L} / r_{a}^{2} \rho_{c} V^{3}$.

To use those results in equation (14) giving the efficiency $\eta_{H}$ for our foil problem, we introduce an equivalent spherical target with equal values for ablation pressure, and mass ablation rate and absorbed power per unit area of ablation surface,

$$
P_{a}^{*}=P_{a}, \quad \dot{m}^{*} / 4 \pi r_{a}^{2}=\dot{m}_{s}, \quad W_{a}^{*} / 4 \pi r_{a}^{2}=I_{a},
$$

quantities marked * corresponding to the spherical problem. If a value for $r_{a}$ is needed we equate the one-sided spherical ablation rate $\dot{m}^{*} / 2$ to the foil rate $\pi r_{f}^{2} \dot{m}_{s}$, so that $r_{a}=2^{-1 / 2} r_{f}$. (The one-sided spherical absorbed power $W_{a}^{*} / 2$ is then equal to the power in the foil problem, $\pi r_{f}^{2} I_{a}$.)

For $\hat{W}_{a}^{*}$ small a deflagration regime exists in spherical geometry (SANZ et al., 1981; Nicolas and SANMARTIN, 1985). We have

$$
\begin{aligned}
\eta_{H} & =0.64 \tilde{\eta}\left(\Delta M_{s} / M_{s 0}\right), & & \hat{\nabla} \rightarrow 0, \\
& =0.34 \bar{\eta}\left(\Delta M_{s} / M_{s 0}\right), & & \hat{\nabla}=0(1),
\end{aligned}
$$

independently of $r_{a}$.

For $\hat{W}_{a}^{*}$ large within the range $10^{2}<\hat{W}_{a}^{*}<10^{5}, Z_{i}$ large and $f$ not too low, results for $P_{a}^{*}\left(W_{a}^{*}\right), \dot{m}^{*}\left(W_{a}^{*}\right)$ are particularly simple. For $f>0.05$ roughly, one has $\dot{m}^{*} / 4 \pi r_{a}^{2}=$ $\beta_{l}^{-1 / 6} \rho_{c} V\left(P_{a}^{*} / \rho_{c} V^{2}\right)^{5 / 6}$ independently of $\hat{V}$ and $\alpha ; \beta_{l} \simeq 11.3$. Also $P_{a}^{*} / \rho_{c} V^{2}=\phi \widehat{W}_{a}^{* 2 / 3}$ where $\phi$ is a weak function of $\hat{W}_{a}^{*}$ and $\hat{V}$, and nearly independent of $\alpha$ and $f\left(\bar{m} / m_{e}\right)^{1 / 2}$ for $f>0.08$ roughly. For $Z_{i}=0(1)$ changes are weak (SANZ et al., 1981; SANZ and SANMARTIN, 1983; NiCOLAS and SANMARTIN, 1985). Experiments with spherical targets suggest that appropriate $f$-values to use in that geometry are sensibly larger than 0.03 (GoLDSACK et al., 1982). For the above conditions we get from (14) with $\Delta M_{s} / M_{s 0}<0.3$,

$$
\begin{aligned}
\eta_{H} & \simeq \frac{P_{a}^{2}}{2 \dot{m}_{s} I_{a}} \times \frac{\Delta M_{s}}{M_{s 0}}=\frac{(4 \pi)^{7 / 9} \beta_{l}^{1 / 6} \phi^{7 / 6} \Delta M_{s}}{2\left(I_{a} / \rho_{c} V^{3}\right)^{2 / 9}} \frac{M_{s 0}}{} \\
& =\frac{9.4 \phi^{7 / 6}}{\hat{W}_{a}^{* 2 / 9}} \frac{\Delta M_{s}}{M_{s 0}},\left(\hat{W}_{a}^{*}=2^{3 / 8} \times 4 \pi\left(U \tau / r_{f}\right)^{3 / 4} \hat{I}_{a}\right) .
\end{aligned}
$$

Note that $\eta_{H} \propto r_{f}^{1 / 6} \propto r_{a}^{1 / 6}$, is weakly dependent on $r_{a}$. We then find (NICOLAS and SANMARTIN, 1985) 


$$
\begin{aligned}
\frac{\eta_{H}}{\Delta M_{s} / M_{s 0}} \simeq \frac{P_{a}^{2}}{2 \dot{m}_{s} I_{a}} \simeq & 0.30(\hat{V} \rightarrow 0) \quad \text { for } \hat{W}_{a}^{*}=10^{2} \\
& 0.20(\hat{V}=0(1)) \\
& 0.06(\hat{V} \rightarrow 0) \\
& \\
& 0.05(\hat{V}=0(1)) \quad \simeq \text { for } \hat{W}_{a}^{*}=10^{5} .
\end{aligned}
$$

The acceleration of thin spherical shells is studied in Appendix B.

\section{DISCUSSION OF RESULTS}

The analysis of Sections 2 and 3 showed that the efficiency $\eta_{H}$ is heavily dependent on the behaviour of the corona, which is parametrized by several dimensionless numbers; mainly $f\left(\bar{m} / m_{e}\right)^{1 / 2}, \hat{I}_{m} \equiv I_{m} / \rho_{c} U^{3}$, and $\hat{U} \equiv \bar{m} U / m_{e} c$, where $f$ is a heat-flux limit factor, $I_{m}$ peak intensity, and $U$ a speed defined by equation (9). This is in agreement with available data, (Fig. 2) from a variety of experiments with thin foils (MCCALL, 1983): it is clear that the data cannot be represented by an universal relation $\eta_{H}\left(\Delta M / M_{0}\right)$. Note also that equation (1), drawn in the figure for comparison, overestimates the efficiency substantially.

Figure 2 shows, in addition, theoretical curves taken from equation (14), using $P_{a}^{2} / 2 \dot{m}_{s} I_{a}$ as given in Table 1 for limit values of the dimensionless parameters (regimes I-IV). For the regimes III and IV the range of validity of the theory is roughly $\Delta M / M_{0}<0.3$ (Section 3); curves have been drawn accordingly. The overall agreement with the data is good. A more detailed comparison would require additional information on each particular experiment.

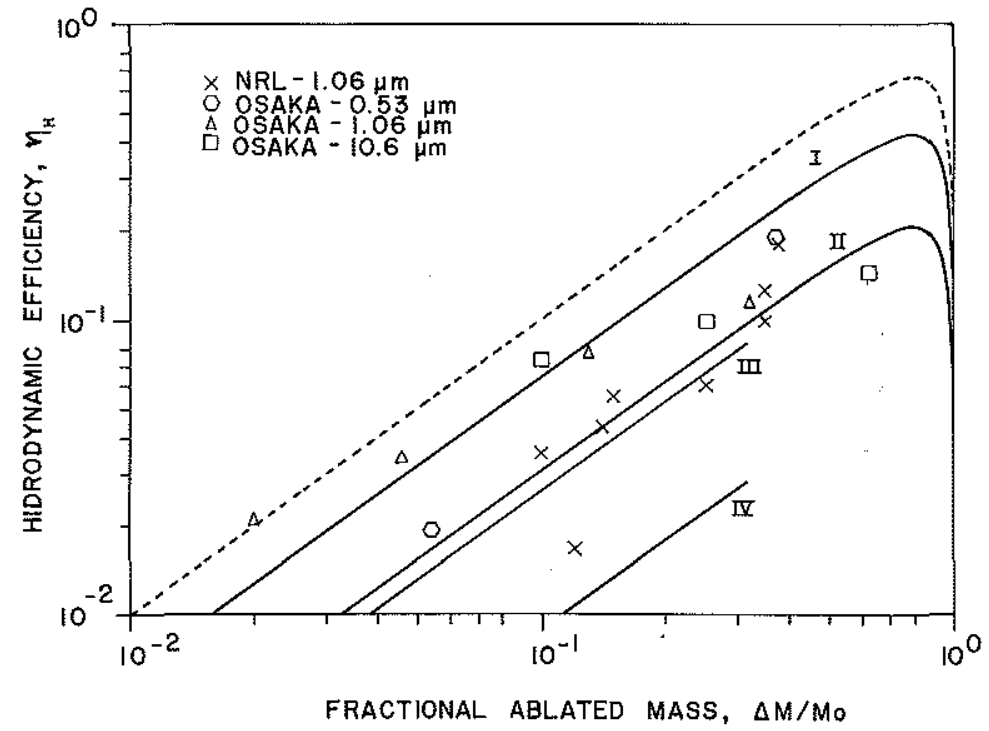

FIG. 2.-Hydrodynamic efficiency $\eta_{H}$ vs ablated mass $\Delta M\left(M_{0}=\right.$ initial foil mass $)$, from both experiments (data points) and theory: the dotted line represents equation (1); lines I-IV represent equation (14) with values from Table 1. 
Table 1 corresponds to a planar corona, or equivalently $U \tau / r_{f}$ small; $\tau$ is the pulse full-width at half-maximum, and $r_{f}$ the spot radius. In the opposite limit, studied in Section 4 in a spherical approximation, a speed $V \equiv U\left(r_{f} / 2^{1 / 2} U \tau\right)^{1 / 4}$ replaces $U$ in dimensionless relations. Results for low intensities, given in equation (17), are similar to those in Table 1 (regimes I and II). Results for high intensities, given in equations (18) and (19), decrease continuously with increasing intensity; they are practically independent of $f$ for the not too low $f$-values suggested by experiments (GOLDSACK et al., 1982).

When $\Delta M$ is not directly measured in an experiment (EIDMANN et al., 1984), it must be calculated if a comparison with theory is to be made. This requires determining the mass ablation rate throughout the pulse: Results on mass ablation rate and ablation pressure will be discussed elsewhere.

The assumption $P_{a}^{2} / 2 \dot{m}_{s} I_{a}=1$, underlying equation (1), is often used, independently of efficiency considerations, to calculate $P_{a}$ from measurements of $\dot{m}_{s}$ and $I_{a}$. Table 1 shows that in this way the ablation pressure may be overestimated by a factor as high as 3 .

Acknowledgement - This research was sponsored by The Comisión Nacional de Investigación CientificoTécnica of Spain and the Instituto de Fusion Nuclear (DENIM) of the Universidad Politecnica de Madrid.

\section{APPENDIX A}

Integrating equation (2) between $x_{v}$, somewhere to the left of the foil where $\rho$ and $P$ vanish, and $x_{a}(t)$, we get

$$
\frac{\mathrm{d}}{\mathrm{d} t} \int_{x_{\nu}}^{x_{a}} \rho \mathrm{d} x-\dot{x}_{a} \rho_{a}=\int_{x_{v}}^{x_{a}} \frac{\partial \rho}{\partial t} \mathrm{~d} x=-\int_{x_{v}}^{x_{a}} \mathrm{~d} x \frac{\partial}{\partial x} \rho v=-\rho_{a} v_{a}
$$

or

$$
\frac{\mathrm{d} M_{s}}{\mathrm{~d} t} \equiv \frac{\mathrm{d}}{\mathrm{d} t} \int_{x_{v}}^{x_{a}} \rho \mathrm{d} x=-\rho_{a}\left(v_{a}-\dot{x}_{a}\right) \equiv-\dot{m}_{s}
$$

which is equation (10).

Integrating equation (3) similarly, we get

$$
M_{s} \frac{\mathrm{d} u}{\mathrm{~d} t}=-P_{a}-\rho_{a}\left(v_{a}-\dot{x}_{a}\right)\left(v_{a}-u\right)
$$

where $u=\int_{x_{v}}^{x_{a}} \rho v \mathrm{~d} x / M_{s}$. Since $P_{a} \sim \rho_{c} v_{\mathrm{exl}}^{2}, \dot{m}_{s} \sim \rho_{\mathrm{c}} v_{\mathrm{ext}}$, and $v_{a} \sim u \sim v_{\mathrm{int}} \ll v_{\mathrm{exl}}$, we may drop the last term in (A.2) to recover equation (11).

Finally integrating (4) with $q=0, l_{\mp}=0$, neglecting $\mathrm{e}_{t n}^{\prime}$ against $\frac{1}{2} v^{2}$ (thin-foil or long-pulse approximation) we get

$$
M_{s} \frac{\mathrm{d} 1}{\mathrm{~d} t} \frac{1}{v^{2}}=-P_{a} v_{a}-\rho_{a}\left(v_{a}-\dot{x}_{a}\right)\left(\frac{1}{2} v_{a}^{2}-\frac{1}{2} \overline{v^{2}}\right)
$$

where $\overrightarrow{v^{2}}=\int_{x_{v}}^{x_{a}} \rho v^{2} \mathrm{~d} x / M_{s}$. Neglecting the last term in (A.3), as in (A.2), we recover (A.3) from (A.2) if $v_{a} \simeq u, \overline{v^{2}} \simeq u^{2}$.

\section{APPENDIX B}

For completeness we consider the acceleration of thin spherical shells (MAX et al., 1983; MAYER et al., 
1983). The equations equivalent to (10) and (11) are

$$
\begin{gathered}
\mathrm{d} M / \mathrm{d} t=-\dot{m}, \\
M \mathrm{~d} u / \mathrm{d} t=-4 \pi r_{a}^{2} P_{a},
\end{gathered}
$$

where $M$ is the mass of the shell at time $t$, and $u$ its outward velocity. For a spherical corona, $f>0.08$, and $10^{2}<\hat{W}_{a} \equiv W_{a} / r_{a}^{2} \rho_{c} V^{3}<10^{5}$, we have $P_{a} / \rho_{c} V^{2}=\phi \hat{W}_{a}^{2 / 3}, \dot{m} / 4 \pi r_{a}^{2} \rho_{c} V=\beta_{l}^{-1 / 6}\left(P_{a} / \rho_{c} V^{2}\right)^{5 / 6}=$ $\beta_{l}^{-1 / 6} \phi^{5 / 6} \hat{W}_{a}^{5 / 9}$ (Section 4). Let $r_{a 0} \equiv r_{a}(t=0)$ and consider a pulse of constant power and duration $\tau$ (Section 3). As the shell collapses, $P_{a}$ and $\dot{m}$ change through their dependence on $r_{a}$; we have

$$
\begin{gathered}
\left(\frac{r_{a 0}}{r_{a}}\right)^{13 / 18} \dot{m}=\dot{m}_{0} \equiv 4 \pi r_{a 0}^{2} \rho_{c} V_{0} \beta_{l}^{-1 / 6} \phi^{5 / 6} \hat{W}_{a 0}^{5 / 9}, \\
\left(\frac{r_{a}}{r_{a 0}}\right)^{4 / 3} P_{a}=P_{a 0} \equiv \rho_{c} V_{0}^{2} \phi \hat{W}_{a 0}^{2 / 3} .
\end{gathered}
$$

From equations (B.1) to (B.4) we obtain

$$
M \frac{\mathrm{d} u}{\mathrm{~d} M}=\frac{4 \pi r_{a 0}^{2} P_{a 0}}{\dot{m}_{0}}\left(\frac{r_{a 0}}{r_{a}}\right)^{1 / 18} .
$$

Neglecting the weak dependence on $r_{a} / r_{a O}$ we get

$$
u \simeq \frac{4 \pi r_{a 0}^{2} P_{a 0}}{\dot{m}_{0}} \ln \frac{M}{M_{0}}, \quad M_{0}=M(t=0)
$$

Using (B.1), (B.3), (B.5), and $u \simeq \mathrm{d} r_{a} / \mathrm{d} t$ we obtain

$$
1-\frac{M}{M_{0}}\left(1-\ln \frac{M}{M_{0}}\right) \simeq \frac{\gamma^{2}}{2}\left(1-\left(\frac{r_{a}}{r_{a 0}}\right)^{31 / 18}\right)
$$

where

$$
\frac{y^{2}}{2}=\frac{18}{31} \frac{\dot{m}_{0}^{2}}{4 \pi r_{a 0} P_{a 0} M_{0}}=\frac{18}{31} \frac{\rho_{c} r_{a 0}}{\rho_{0} \Delta R} \frac{\phi^{2 / 3}}{\beta_{!}^{1 / 3}} \hat{W}_{a 0}^{4 / 9} .
$$

One can show that velocities in the corona are of order $V_{0} \hat{W}_{a 0}^{1 / 9}$. Hence, the corona will be quasirsteady as long as $u \ll V_{0} \hat{W}_{a 0}^{1 / 9}$. According to (B.3)-(B.5), we have $u \simeq\left(\beta_{l} \phi\right)^{1 / 6} V_{0} \hat{W}_{a 0}^{1 / 9} \ln \left(M / M_{0}\right)$ so that the above inequality is equivalent to $\Delta M / M_{0} \equiv 1-M / M_{0} \ll 1$, as in the planar case. Equation (B.6) shows that this condition is satisfied for the entire implosion $\left(0<r_{a}<r_{a 0}\right)$ if $\gamma^{2} / 2$ is small. This is fortunately the usual case in experiments: for instance, if $\Delta R=r_{a 0} / 20, \rho_{c} / \rho_{0}=1.3 \times 10^{-3}$ and $m_{i} / Z_{i} \simeq 2 m_{p}$ (Al shell, $1.06 \mu \mathrm{m}$ light, fully ionised corona), $r_{a 0}=200 \mu \mathrm{m}$, and $W_{a}=10^{12} \mathrm{~W}$, we have $\gamma^{2} / 2 \simeq 0.03\left(\hat{W}_{a 0} \simeq 2.9 \times 10^{3}, \phi \simeq 0.1\right)$. (For $\gamma^{2} / 2$ large, the shell would be ablated with almost no collapse.) Then equation (B.6) takes the form

$$
\left(\Delta M / \gamma M_{0}\right)^{2}=1-\left(r_{a} / r_{a 0}\right)^{31 / 18} .
$$

Using (B.8) in (B.1) we get

$$
\tau=\frac{\gamma M_{0}}{\dot{m}_{0}} I\left(\frac{\Delta M}{\gamma M_{0}}\right), \quad I(s)=\int_{0}^{s} \frac{\mathrm{d} s^{\prime}}{\left(1-s^{\prime 2}\right)^{1 / 31}} .
$$

Equations (B.8) and (B.9) determine the values of $r_{a}$ and $\Delta M$ at the end of the pulse, as long as (B.9) gives $\Delta M / \gamma M_{0}<1$; for longer pulses, equation (B.9) with $\Delta M / \gamma M_{0}=1$ gives the time for total collapse $\left(r_{a}=0\right)$.

Equations (B.5) and (B.9) lead to

$$
\eta_{H} \equiv \frac{M u^{2}}{2 W_{a} \tau}=\frac{\left(4 \pi r_{a 0}^{2} P_{a 0}\right)^{2}}{2 W_{a} \dot{m}_{0}} \frac{\Delta M}{M_{0}} \frac{\Delta M / \gamma M_{0}}{I\left(\Delta M / \gamma M_{0}\right)}
$$


where we set $\bar{\eta}\left(\Delta M / M_{0}\right) \simeq \Delta M / M_{0}(<0.2$ or 0.3$)$. When compared with

$$
\eta_{H}=\frac{2 \pi r_{a 0}^{2} P_{u 0}^{2}}{\dot{m}_{0} W_{t} / 4 \pi r_{a 0}^{2}} \bar{\eta}\left(\frac{\Delta M}{M_{0}}\right)
$$

(MAX et al., 1983) three differences appear: (i) The full expression $\bar{\eta}\left(\Delta M / M_{0}\right)$ was retained in (B.11). Since a quasisteady corona was also assumed to get (B.11), it should not be valid above $\Delta M / M_{0}=0.3$, say, and thus $\bar{\eta} \simeq \Delta M / M_{0}$. Secondly, MAX et al. (1983) wrote $\tau=\Delta M / \dot{m}_{0}$, instead of (B.9), missing the factor $\left(\Delta M / \gamma M_{0}\right) / I\left(\Delta M / \gamma M_{0}\right)$. For $0<s<1$ we have $1>s / I>0.70$; hence (B.11) may overestimate the efficiency by as high as $42 \%$.

Finally, approximate results for the corona for small $f$ (MAX et al., 1980; SANZ, and SANMARTIN, 1983) were used in (B.11). For the not too low $\int$ suggested previously (SANZ and SANMarTin, 1983) we have (B.3) and (B.4), and equation (B.10) becomes

$$
\eta_{H}=\frac{2 \pi \beta_{l}^{1 / 6} \phi^{7 / 6}}{\hat{W}_{a 0}^{2 / 9}} \frac{\Delta M}{M_{0}} \frac{\left(\Delta M / \gamma M_{0}\right)}{I\left(\Delta M / \gamma M_{0}\right)}
$$

which is just $(18) \times\left(\Delta M / \gamma M_{0}\right) / I\left(\Delta M / \gamma M_{0}\right)$. Using now (B.7) we finally get

$$
\eta_{H}=\left(2 \pi\left(\frac{36}{31} \frac{\rho_{c} r_{a 0}}{\rho_{0} \Delta R}\right)^{1 / 2} \phi^{3 / 2}\right) \times \frac{\left(\Delta M / \gamma M_{0}\right)^{2}}{I\left(\Delta M / \gamma M_{0}\right)} .
$$

For $\rho_{c} / \rho_{0}=1.3 \times 10^{-3}, r_{a 0} / \Delta R=20, \phi=0.1$, the expression within the bracket is 0.02 ; the function $s^{2} / I(s)$ has a maximum 0.76 at $s \simeq 0.94$. Thus $\eta_{H}$ (maximum) $\simeq 0.015$.

\section{REFERENCES}

Aranas'ev Yu.V., Gamalit E. G., Krokhin O. N. and Rozanov V. B. (1977) Soviet Phys. JeTP 44, 311. ANISIMOV S. I. (1970) JETP Lett. 12, 287.

Barrero A. and Sanmartin J. R. (1977) Physics Fluids 20, 1155.

Barrero A. and Sanmartin J. R. (1980) Plasma Physics 22, 617.

Duderstadt J. J. and Moses G. A. (1982) Inertial Confinement Fusion, Wiley, New York.

Eudmann K., Amiranoff F., Fudosejgys R., Maaswinkel A. G. M., Petsch R., Sigel R., Spindler G., Yung-Lu Teng, Tsakiris G. and WrTKowski S. (1984) Report MPQ 84, Max-Planck-Institut Für Quantenoptik, D-8046 Garching, F.R.G.

EstabroOK K. and KRUER W. L. (1978) Phys. Rev. Lett. 40, 42.

Fabbro R. (1982) Doctoral Thesis, Université de Paris Sud., Centre d'Orsay.

Felber F, S. (1977) Phys. Rev. Lett. 39, 84.

Forslund D. W., Kindel J. M. and LeE K. (1977) Phys. Rev. Lett. 39, 284.

Gitomer S. J., Morse R. L. and Newbergler B. S. (1977) Physics Fluids 20, 234.

Goldsack T. J., Kilkenny J. D., MacGowan B. J., Cunningham P. F., Lewis C. L. S., Key M. H. and

Rumsiy P. T. (1982) Physics Fluids 25, 1634.

Johnston T. W. and Dawson J. M. (1973) Physics Fluids 16, 722.

MaX C. E., MCKeE C. F. and MeAd W. C. (1980) Physies Fluids 23, 1620.

MaX C. E., Lindl J. D. and MEAd W. C. (1983) Nucl. Fusion 23, 131.

Mayer F. J., Larsen J. T. and Steele J. W. (1983) Physics Fluids 26, 830.

MCCAll G. H. (1983) Plasma Physics 25, 237.

Nicolas J. A. (1984) Doctoral Thesis, Universidad Politécnica de Madrid.

Nicolas J. A. and Sanmartin J. R. (1985) Plasma Physics in press.

Ramis R. and Sanmartin J. R. (1983) Nucl. Fusion 23, 739.

Ripin B. H., Decoste R., Obenschain S. P., Bodner S. E., Mclean E. A., Young F. C., Whitlock R. R., Armstrong C. M., Grun J., Stamper J. A., Gold S. H., Nagel D. J., Lehmberg R. H. and MCMAHON J. M. (1980) Physics Fluids 23, 1012.

SANMARTin J. R. and Bakrero A. (1978a) Physics Fluids 21, 1957.

SANMArTin J. R. and BArrero A. (1978b) Physics Fluids 21, 1967.

Sanmartin J. R., Montañes J. L. and Barrero A. (1983) Physics Fluids 26, 2754.

Sanz J., Liñan A., Rodrigutz. M. and Sanmartin J. R. (1981) Physics Fluids 24, 2098.

SAnZ J. and SANMARTin J. R. (1983) Physics Fluids 26, 3361.

Spitzer L. (1967) Physics of Fully Ionised Gases, Wiley, New York.

Zel'Dovich Ya.B. and RaIzer Yu.P (1967) Physics of Shock Waves and High-temperature Hydrodynamic

Phenomena, Academic Press, New York. 